\title{
Vitamin D status influences cytokine production and MALAT1 expression from the PBMCs of patients with coronary artery disease and healthy controls
}

\author{
Peyman Nowrouzi-Sohrabi, ${ }^{\mathbf{1}}$ \\ (iD) Mehdi Kalani ${ }^{3}$ \\ (iD) Peyman Izadpanah ${ }^{4}$ \\ (iD) Hassan Ahmadvand ${ }^{5,6}$ \\ (iD Masoumeh Fakhour ${ }^{7}$ \\ (iD) Reza Fadaei ${ }^{8}$ \\ Deghdad Khorshidifar ${ }^{4}$ \\ (iD) Atefeh Seghatoleslami,9
}

\begin{abstract}
1. Department of Biochemistry, School of Medicine, Shiraz University of Medical Sciences, Shiraz, Iran. 2. Student Research Committee, Shiraz University of Medical Sciences, Shiraz, Iran. 3. Professor Alborzi Clinical Microbiology Research Center, Shiraz University of Medical Sciences, Shiraz, Iran. 4. Cardiology Department, Shiraz University of Medical Sciences, Shiraz, Iran. 5. Department of Biochemistry, Faculty of Medicine, Lorestan University of Medical Sciences, Khorramabad, Iran. 6. Razi Herbal Researches Center, Lorestan University of Medical Sciences, Khorramabad, Iran. 7. Faculty of Allied Medical Sciences, Zabol University of Medical Sciences, Zabol, Iran.

8. Sleep Disorders Research Center, Kermanshah University of Medical Sciences, Kermanshah, Iran.
\end{abstract} 9. Research Center for Traditional Medicine and History of Medicine, Medical School, Shiraz University of Medical Sciences, Shiraz, Iran.

http://dx.doi.org/10.1590/1806-9282.66.12.1712

\section{SUMMARY}

OBJECTIVE: This study aimed to investigate the long non-coding RNA metastasis-associated lung adenocarcinoma transcript 1 (IncRNA MALAT1) expression and its role in cytokine production from peripheral blood mononuclear cells (PBMCs) in patients with coronary artery disease (CAD) and non-CAD participants (NCAD).

METHODS: Blood samples were taken from 15 patients with CAD and 15 NCAD individuals. The plasma was used for biochemical analyses. MALAT1 and CD36 expressions were evaluated in the isolated peripheral blood mononuclear cells (PBMCs) by real-time $P C R$. Furthermore, the levels of inflammatory cytokines e.g. interleukin (IL)-6, IL-10, and IL-22 were measured in the supernatants of the cultured PBMCs by flow cytometry.

RESULTS: The levels of MALAT1 and CD36 were not significantly different between the CAD and NCAD groups. However, a lower level of MALAT1 and CD36 was observed in PBMCs of vitamin D deficient $(<15 \mathrm{ng} / \mathrm{ml})$ CAD and NCAD participants. Furthermore, the vitamin D deficient $(<15 \mathrm{ng} / \mathrm{ml}$ ) group showed a significantly higher plasma level of IL-6, IL-10, and IL-22 compared to the non-deficient ( $\geq 15 \mathrm{ng} /$ $\mathrm{ml}$ ) group. In addition, significant positive correlations were found between CD36, IL-22, and fasting blood sugar (FBS) with MALAT1.

CONCLUSION: Given that in vitamin D deficient individuals a decreased level of MALAT1 was associated with CD36 expression and increased IL-22 production, vitamin D supplementation may play a role in reducing MALAT1/CD36/IL-22 mediated complications such as T2DM and CAD, especially in vitamin D deficiency.

KEYWORDS: Vitamin D. CD36 antigens. Interleukins. Coronary artery disease. RNA, long noncoding.

DATE OF SUBMISSION: 30-Jul-2020

DATE OF ACCEPTANCE: 08-Aug-2020

CORRESPONDING AUTHOR: Atefeh Seghatoleslam

Department of Biochemistry, School of Medicine, Shiraz University of Medical Sciences, Shiraz, Iran - P. O. Box: 71348-45794

Tel: +98 917 319-7091 / Fax: +98 71 3230-3029

E-mail: seghatolea@sums.ac.ir 


\section{INTRODUCTION}

Vitamin D deficiency is associated with both calcium and bone metabolism disorders, as well as vascular and low-grade inflammatory disease ${ }^{1,2}$. Some long non-coding RNAs (lncRNAs), such as lncRNAs HOTAIR and $\mathrm{H} 19$, have been found to be regulated by vitamin D through vitamin D receptors in multiple cancers. LncRNAs are transcripts, longer than 200 nucleotides without protein-coding ability, which are involved in both transcriptional and post-transcriptional regulation, and therefore, in the pathophysiology of diseases such as coronary artery disease (CAD) ${ }^{3}$. Amongst the reports, lncRNA metastasis-associated lung adenocarcinoma transcript 1 (MALAT1), also known as NEAT2, might protect against the occurrence of CAD and alleviate inflammation in atherosclerosis. The role of the scavenger receptors $\mathrm{B} 1$ or $\mathrm{CD} 63$ as receptors for oxidized low-density lipoproteins (ox-LDL) is vital in foam cell formation, which is a critical step in the development of atherosclerosis. Moreover, a recent in-vitro study reported the influence of MALAT1 on the expression of CD36 ${ }^{4}$.

Previously, the role of inflammatory cytokines has been well evaluated. Interleukin (IL)-22, a member of the IL-10 related cytokine superfamily, has a dual role in inflammation. Gong et al. ${ }^{5}$ suggested IL-22 functions as a double-edged sword in type 2 diabetes mellitus (T2DM) and CAD.

Consequently, given the inflammatory nature of atherosclerosis and the role of MALAT1 and CD36 in atherosclerosis, the present study aimed to assess MALAT1/CD36 levels as well as cytokines, such as IL-6, IL-10, and IL-22 in CAD patients compared to healthy controls. However, little is known about the regulation and functions of lncRNAs in the treatment and prevention of cardiovascular diseases with vita$\min \mathrm{D}$.

\section{METHODS}

\section{Participants}

A cross-sectional study included 15 CAD patients and 15 non-CAD (NCAD) or healthy individuals who were admitted to the Al-Zahra Heart Hospital, Shiraz, Iran, from October 2018 until January 2019, requiring coronary angiography (CA) or CT angiography (CTA). Based on the CA and CTA results, the study population was divided into CAD and NCAD groups. CAD was diagnosed by a cardiologist if there was more than $50 \%$ stenosis in at least one coronary artery.
The serum level of 25-hydroxy vitamin D was measured in these two groups and classified as deficient $(<15 \mathrm{ng} / \mathrm{mL})$ and non-deficient $(\geq 15 \mathrm{ng} / \mathrm{mL})^{6,7}$. Patients with diabetes mellitus, malignancies, infections, blood diseases, and chronic renal or liver failure, history of inflammatory diseases, and those using immunosuppressive drugs were excluded from the study. Demographic and anthropometric data including age, body mass index (BMI), as well as data regarding medication usage by patients were collected via a questionnaire, and using the hospital patient records.

\section{Laboratory assessments}

The biochemical characteristics including fasting blood sugar (FBS), lipid profile, and liver enzyme activities were measured using commercially enzymatic kits by BS 200 autoanalyzer (Mindray, China). Vitamin D was measured using the HPLC method by Tosoh G8 instrument (Tosoh, USA).

\section{PBMCs isolation and cell culture}

PBMCs were isolated from $10 \mathrm{ml}$ peripheral blood samples by centrifugation over Ficoll-Hypaque gradients (Lymphodex, InnoTrain, Germany). The cells were washed, using RPMI 1640 medium and resuspended in RPMI-1640 medium supplemented with $50 \mathrm{U} / \mathrm{mL}$ penicillin, $50 \mu \mathrm{g} / \mathrm{mL}$ streptomycin, and $10 \%$ fetal bovine serum. In order to evaluate the MALAT1 expression level, $2 \times 10^{6}$ cells were incubated for $5 \mathrm{~h}$ at $5 \% \mathrm{CO}_{2}, 95 \%$ humidity, and $37^{\circ} \mathrm{C}$. Simultaneously, additional $10^{5}$ cells were incubated for $48 \mathrm{~h}$ to assess the cytokines level; hence, supernatants were collected and stored at $-80{ }^{\circ} \mathrm{C}$ until further analysis.

\section{RNA extraction and cDNA synthesis}

Total RNA was extracted from $2 \times 10^{6}$ cells, using the Trizol reagent (Bio Basic Inc., Canada) and then converted into cDNA in reverse transcription reactions using Thermo Scientific RevertAid First Strand cDNA Synthesis Kit (Thermo Scientific, USA) based on the manufacturer's instructions.

\section{Quantitative Real-Time PCR}

Equal amounts of cDNA for each sample were amplified using RealQ Plus 2x Master Mix Green (Ampliqon, Denmark) and specific primers for MALT1, CD36, and beta-actin as a reference gene, based on the manufacturer's protocol. The relative expressions were calculated using $2^{-\mathrm{ACT}}$ method. 


\section{Cytokine assay}

The supernatants of the cultured PBMCs were used to assess the IL-6, IL-10, and IL-22 levels. Cytokines were measured by LEGENDplex ${ }^{\mathrm{TM}}$ Human Th22 Panel (Biolegend, USA) using a flow cytometer (FACS Calibur, $\mathrm{BD}, \mathrm{USA})$, according to the manufacture's instruction.

\section{Statistical analysis}

Statistical analysis was performed using SPSS 22.0 (IBM Inc., USA) and GraphPad Prism version 8.2 (San Diego, CA, USA). The categorical data were tested by Fisher exact test and presented by frequency and percentile. Continuous variables are presented as mean \pm standard error of the mean (SEM) and tested by the Mann-Whitney U test and one-way ANOVA. The Spearman correlation test was used to determine the relationship between the variables. A P value $<0.05$ was considered statistically significant.

\section{RESULTS}

Clinical and laboratory findings of the participants

The demographic and clinical characteristics of participants are shown in Table 1. Hip circumference, echocardiogram ejection fraction, and diastolic blood pressure were significantly lower in patients with CAD compared to NCAD. Furthermore, body mass index, hip circumference, and AST activity were significantly higher in the vitamin D deficient group compared to the non-deficient. For a detailed description, see Table S1 in Supplementary Materials.

The comparison of MALAT1 and CD36 levels between the investigated groups

We first analyzed MALAT1 and CD36 in the PBMCs of CAD and NCAD individuals. We found that there was no significant difference between the CAD and NCAD groups ( $p=0.238$ and $p=0.945)$ (Fig $1 A$ and $D)$. Interestingly, after classification into non-deficient and deficient groups, we noticed that MALAT1 and CD36 expressions were significantly lower when there was vitamin D deficiency (Fig 1B and 1E) ( $p=0.015$ and $\mathrm{p}=0.022$ ). In addition, the comparison of the MALAT1 levels between the CAD and NCAD groups based on the vitamin D status showed that MALAT1 expression was significantly higher in the NCAD non-deficient group compared to the NCAD deficient group $(p=0.023)$. However, the difference between CAD non-deficient and deficient groups was not statistically significant (Fig 1C).

FIGURE 1

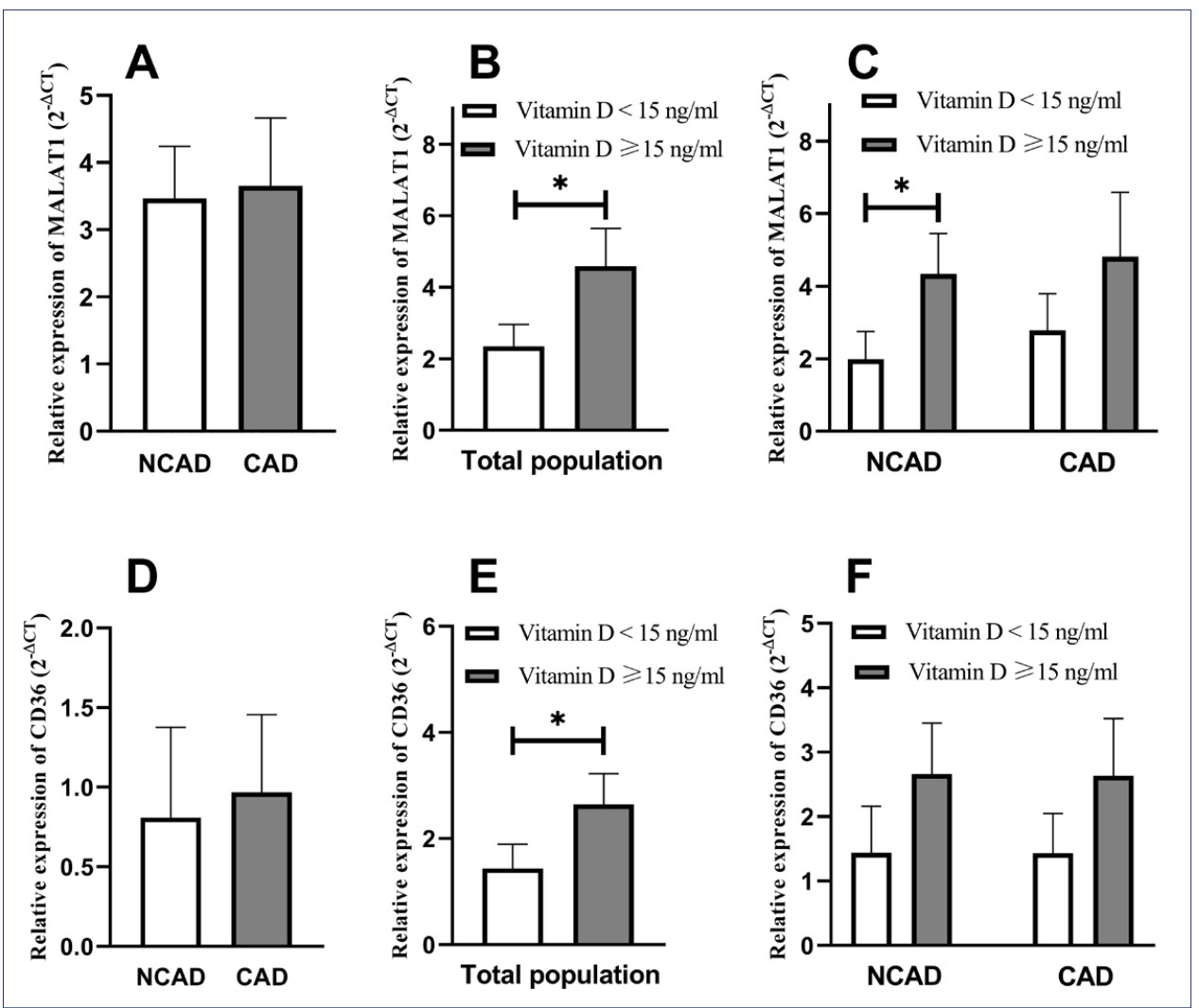


Comparing the cytokine production levels between the study groups

The ex-vivo cytokine profiles produced by PBMCs are depicted in Fig 2. The results showed that the production of IL-6, IL-10, and IL-22 were significantly higher in the vitamin D deficient group compared to the non-deficient group $(p=0.006, p=0.014$, and $p=0.018$, respectively) (Fig $2 A, C$ and $E$ ). When CAD and NCAD individuals were divided based on the vitamin D status, the cytokines levels were significantly higher in the $\mathrm{CAD}$ deficient group compared to that in the CAD non-deficient group ( $\mathrm{p}=0.021, \mathrm{p}=0.009$, and $\mathrm{p}=0.040$, respectively) (Fig 2B, D, and F).

\section{Association between clinical and biochemical characteristics with MALAT1}

As presented in Table 2, the spearman correlation test revealed a significant correlation between the

\section{FIGURE 2}

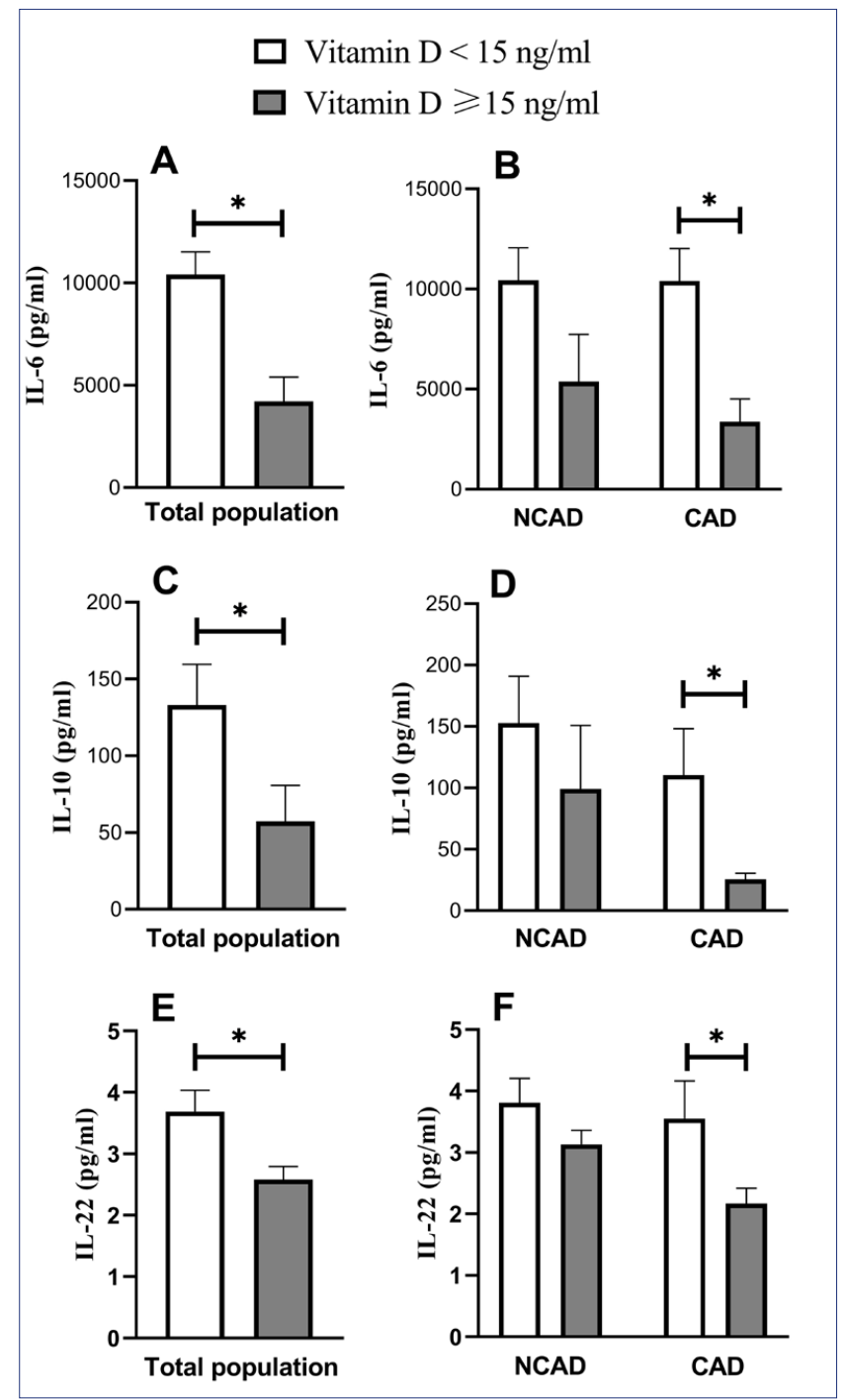

MALAT1 and CD36 expressions in the PBMCs of the studied groups. In order to evaluate the association of MALAT1 expression with cytokine production from PBMCs, we analyzed the correlation between MALAT1 expression and cytokine level. MALAT1 showed a significant negative correlation with IL-22 in the total population of the CAD and NCAD groups $(r=-0.406$, $\mathrm{p}=0.036)$. There were no significant correlations between MALAT1 and other cytokines. In addition, MALAT1 expression in PBMCs of the NCAD group correlated directly and indirectly with FBS ( $\mathrm{r}=0.606$, $\mathrm{p}=0.022)$ and vitamin $\mathrm{D}(\mathrm{r}=-0.693, \mathrm{p}=0.006)$. For a detailed description, see Table S2 in the Supplementary Materials.

\section{DISCUSSION}

In this study, we observed that the relative expression of MALAT1 and CD36 were comparable between CAD patients and healthy controls. However, dysregulated expression of MALAT1 and CD36 has been reported in different pathological conditions. The unregulated expression of MALAT1 was reported from the PBMCs of type 2 diabetics and patients with $\mathrm{MI}^{8}$. However, the function of MALAT1 in inflammation related to vitamin $\mathrm{D}$ status and CAD remains unknown. Accordingly, this study investigated the effects of vitamin D status on the expression of MALAT1 as well as cytokines IL-6, IL-10, and IL-22 from the PBMCs of the patients with CAD as well as NCAD individuals.

The results revealed that although MALAT1/CD36 expression was higher in the CAD group, upregulated levels of MALAT1/CD36 were not significantly associated with CAD. A similar finding to our study was reported by Toraih et al. ${ }^{9}$, who reported that MALAT1 levels, the median MALAT1 expression in the PBMCs of patients with previous cardiac events was comparable with those of control groups. However, MALAT1 is identified and characterized as a regulator of inflammatory response via TLR4/ TLR4/NF- $\mathrm{KB}^{8}$.

Some controversy still exists regarding CD36 expression levels in $\mathrm{CAD}^{10,11}$. In the present study, CD36 was highly correlated with MALAT1. According to previous studies, it is suggested that MALAT1 might be a contributing factor to CD36 upregulation in the PBMCs of CAD patients. In this line, Huangfu et al. ${ }^{4}$ reported that MALAT1 through the accumulation of beta-catenin on the CD36 promoter raises CD36 transcription. 
Interestingly, we observed that the level of MALAT1 was significantly lower in the PBMCs of vitamin D deficient individuals compared to that in non-deficient ones. To the best of our knowledge, this is the first study to investigate the association between vitamin D status and MALAT1 expression. Based on these results, regardless of the CAD status, MALAT1 expression is increased in the PBMCs of vitamin D deficient individuals. The molecular mechanisms underlying increased expression of MALAT1 in the non-deficient group are not well understood; however, the anti-inflammatory effect of vitamin D can be a determining factor. In several studies, the association between MALAT1 and inflammation has been reported ${ }^{8}$. However, the protective effects of vitamin D on colorectal cancer through the modulation of some lncRNAs have been previously suggested ${ }^{12}$.

Accordingly, the upregulation of MALAT1 could be proposed as a contributing factor to the anti-inflammatory effects of vitamin D. As a detailed description, regarding the results, vitamin D deficiency was significantly associated with a higher production level of cytokines including IL-6 (2.5 fold), IL-10 (2.3 fold), IL-22 (1.4 fold) compared to the non-deficient group (Fig 2). The effects of vitamin D on the level of inflammatory cytokines have already been investigated. Vitamin D might inhibit the production of pro-inflammatory cytokines (IL-6 and TNF- $\alpha$ ) via directly inhibiting the

\section{FIGURE 3}

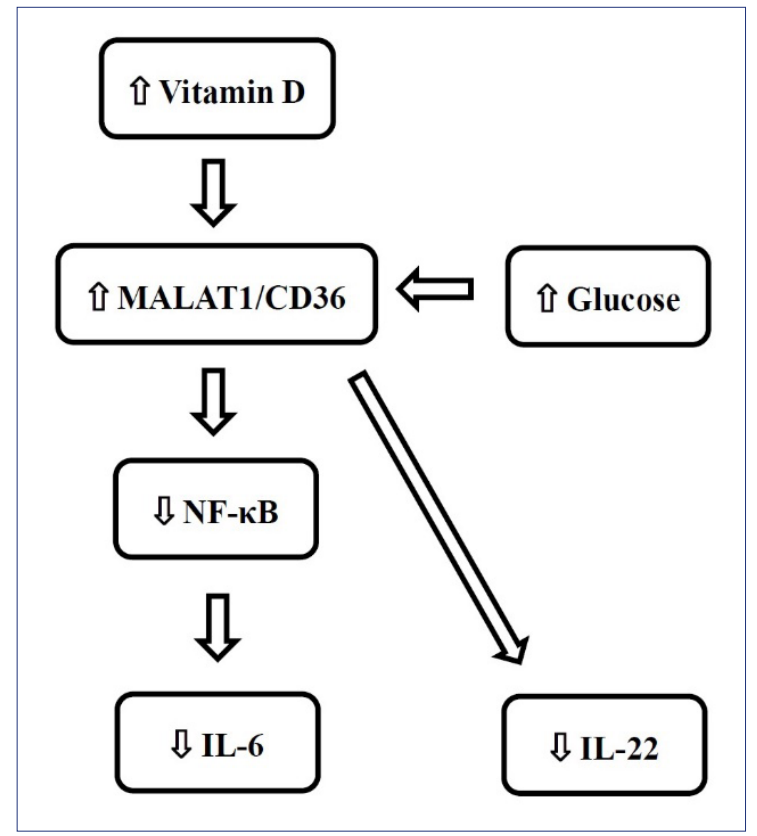

NF- $k$ B signaling pathway (Fig 3$)^{13}$. Importantly, in this study, a significant negative correlation was also found between MALAT1 and IL-22.

Furthermore, based on the present and previous studies, we found that FBS increases the expression of MALAT1 in the PBMCs of CAD patients ${ }^{14}$. In addition, MALAT1 expressions were negatively correlated with the production of IL-22. To the best of our knowledge, this is the first study to investigate the association between MALAT1 and IL-22. The molecular mechanisms underlying this correlation are not well understood; however, different factors might be involved, such as hyperglycemia, especially because a significant direct correlation has also been observed between IL-22 and FBS (Fig 3). Furthermore, the increased level of IL-22 in the vitamin D deficient group might be partly through MALAT1 (Fig 3).

So far, few studies have been performed on the role of IL-22 in CAD. Shen et al. ${ }^{15}$ reported that plasma concentrations of IL-22 are decreased in impaired fasting glucose (IFG) and T2DM patients, and decreased plasma concentrations of IL-22 is an independently susceptible factor for IFG and T2DM. Decreased plasma IL-22 levels increase the morbidity of diabetes.

In this line, Puthanveetil et al. ${ }^{14}$ incubated human umbilical vein endothelial cells with high glucose levels, which led to an increase in MALAT1 expression after 12h. IL-22 protects endothelial cells from glucose-induced injury. All these findings suggest that the MALAT1 correlation with IL-22 might be a potential target for the treatment of chronic inflammatory diseases, such as T2DM and $\mathrm{CAD}^{5}$.

\section{CONCLUSION}

The results of the present study suggest that MALAT1 expression is decreased in the PBMCs of the vitamin D deficient group, and this decreased expression appears to be associated with CD36 expression and increased IL-22 production from PBMCs. The protective effects of IL-22 against hyperglycemia and the observed correlation of MALAT1 with CD36 and FBS in the CAD group suggest that MALAT1/CD36/ IL-22 might be also potential targets, using vitamin D supplementation for treating vascular complications mediated by hyperglycemia in T2DM and CAD especially in vitamin D deficiency. 


\section{Funding}

The present article was extracted from the thesis written by P N-S and was financially supported by the vice chancellery for research in the Shiraz University of Medical Sciences (grant No. 1396-01-01-16594) and granted partly by Professor Alborzi, Clinical Microbiology Research Center (grant No. 96-7).

\section{Compliance With Ethical Standards}

This study was approved by the local Ethics Committee of the Shiraz University of Medical Sciences (IR.SUMS.REC.1397.687). After explaining the study objectives, written informed consent was obtained from the participants.

\section{Conflict of Interests}

The authors have no conflicts of interest to declare.

\section{Author's Contribution}

P.N: Performed all experiments, analyzed the data, and wrote the manuscript. P.I., M.Kh.: Analyzed the CA and CTA reports. P.N., H.A., R.F.: Contributed to concept and design. M.F.: Contributed to sampling and experiments. A.S., M.Ka: Contributed to the concept and design, financial support, supervising practical performances, and final approval of the manuscript. All authors read and approved the final manuscript.

\section{RESUMO}

OBJETIVO: O objetivo deste estudo foi investigar a expressão do RNA longo não codificante IncRNA MALAT1 e o seu papel na produção de citocinas a partir de células mononucleares do sangue periférico (PBMCS) em pacientes com doença arterial coronariana (DAC) e participantes sem DAC (NDAC).

MÉTODOS: Amostras de sangue foram coletadas de 15 pacientes com DAC e 15 indivíduos NCAD. O plasma foi usado para análises bioquímicas. As expressões de MALAT1 e CD36 foram avaliadas nas células mononucleares do sangue periférico (PBMCS) isoladas por PCR em tempo real. Além disso, os níveis de citocinas inflamatórias, como a interleucina (IL)-6, IL-10 e IL-22 foram medidas no sobrenadante da cultura de PBMCs por citometria de fluxo.

RESULTADOS: Os níveis de MALAT1 e CD36 não foram significativamente diferentes entre os grupos DAC e NDAC. No entanto, um nível inferior de MALAT1 e CD36 foi observado nas PBMCs de participantes com deficiência de vitamina D ( $15 \mathrm{ng} / \mathrm{ml})$ tanto no grupo DAC quanto no NDAC. Além disso, o grupo com deficiência de vitamina $D(<15 \mathrm{ng} / \mathrm{ml})$ apresentou um nível plasmático significativamente maior de IL-6, IL-10 e IL-22 em comparação com o grupo sem a deficiência ( $\geq 15 \mathrm{ng} / \mathrm{ml})$. Além disso, foram encontradas correlações positivas significativas entre CD36, IL-22, e glicemia de jejum (GJ) e o MALAT1.

CONCLUSÃo: Dado que em indivíduos com deficiência de vitamina D a diminuição do nível de MALAT1 foi associada com a expressão de CD36 e produção aumentada de IL-22, a suplementação de vitamina D pode ter um papel importante na redução de complicações mediadas por MALAT1/CD36/IL-22, tais como DMT2 e DAC, especialmente em casos de deficiência de vitamina D.

PALAVRAS-CHAVE: Vitamina D. Antígenos CD36. Interleucinas. Doença da artéria coronariana. RNA longo não codificante.

\section{REFERENCES}

1. Erkus E, Aktas G, Kocak MZ, Duman TT, Atak BM, Savli H. Diabetic regulation of subjects with type 2 diabetes mellitus is associated with serum vitamin D levels. Rev Assoc Med Bras. 2019;65(1):51-5.

2. Bonatto S, Paniz VMV, Dutra CF, Henn RL. Vitamin D serum levels and peripheral arterial disease among Southern Brazilian adults. Rev Assoc Med Bras. 2020;66(3):268-74.

3. Xiong $G$, Jiang $X$, Song $T$. The overexpression of $I n c R N A H 19$ as a diagnostic marker for coronary artery disease. Rev Assoc Med Bras. 2019;65(2):110-7.

4. Huangfu N, Xu Z, Zheng W, Wang Y, Cheng J, Chen X. LncRNA MALAT1 regulates oxLDL-induced $C D 36$ expression via activating $\beta$-catenin. Biochem Biophys Res Commun. 2018;495(3):2111-7.

5. Gong F, Wu J, Zhou P, Zhang M, Liu J, Liu Y, et al. Interleukin-22 might act as a double-edged sword in type 2 diabetes and coronary artery disease. Mediators Inflamm. 2016;2016:8254797.

6. Musavi Mehdiabadi F, Ahmadi F, Lesan Pezeshki M, Razeghi E. The relationship between serum level of 25-hydroxy vitamin $D$ and cytomegalovirus infection in kidney transplant recipients. Iran J Kidney Dis. 2019;13(4):225-31.

7. Ahmadi F, Damghani S, Lessan-Pezeshki M, Razeghi E, Maziar S, Mahdavi-Mazdeh M. Association of low vitamin D levels with metabolic syndrome in hemodialysis patients. Hemodial Int. 2016;20(2):261-9.

8. Yan Y, Song D, Song X, Song C. The role of IncRNA MALAT1 in cardiovascular disease. IUBMB Life. 2020;72(3):334-42.
9. Toraih EA, El-Wazir A, Alghamdi SA, Alhazmi AS, El-Wazir M, Abdel-Daim MM, et al. Association of long non-coding RNA MIAT and MALAT1 expression profiles in peripheral blood of coronary artery disease patients with previous cardiac events. Genet Mol Biol. 2019;42(3):509-18.

10. Munjas I, Sopić M, Spasojević-Kalimanovska V, Kalimanovska-Oštrić D, Andelković K, Jelić-Ivanović Z. Association of adenylate cyclase-associated protein 1 with coronary artery disease. Eur J Clin Invest. 2017;47(9):659-66.

11. Paulsson JM, Held C, Jacobson SH, Lundahl J. In vivo extravasated human monocytes have an altered expression of CD16, HLA-DR, CD86, CD36 and CX(3)CR1. Scand J Immunol. 2009;70(4):368-76.

12. Zhu $Y$, Chen $P, G a o Y, T a N$, Zhang $Y$, Cai I, et al. MEG3 activated by vitamin $D$ inhibits colorectal cancer cells proliferation and migration via regulating clusterin. EBioMedicine. 2018;30:148-57.

13. Al-Rasheed NM, Al-Rasheed NM, Bassiouni YA, Hasan IH, Al-Amin MA, Al-Ajmi HN, et al. Vitamin D attenuates pro-inflammatory TNF- $\alpha$ cytokine expression by inhibiting NF-kB/p65 signaling in hypertrophied rat hearts. J Physiol Biochem. 2015;71(2):289-99.

14. Puthanveetil $P$, Chen S, Feng $B$, Gautam A, Chakrabarti S. Long non-coding RNA MALAT1 regulates hyperglycaemia induced inflammatory process in the endothelial cells. J Cell Mol Med. 2015;19(6):1418-25.

15. Shen |, Fang Y, Zhu H, Ge W. Plasma interleukin-22 levels are associated with prediabetes and type 2 diabetes in the Han Chinese population. J Diabetes Investig. 2018;9(1):33-8. 